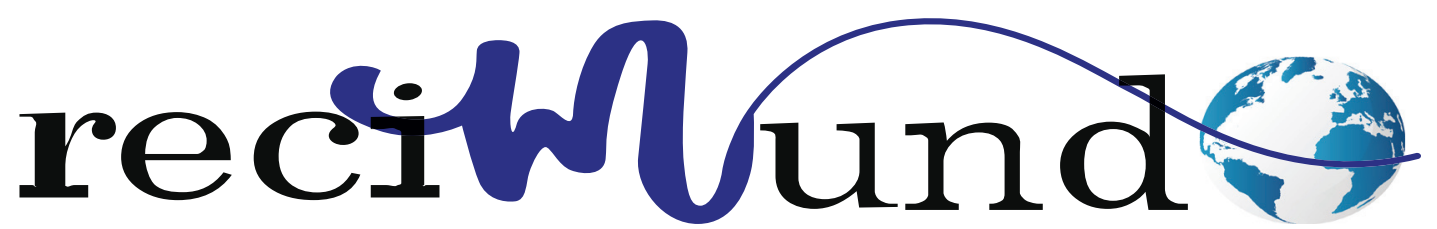

Revista Científica Mundo de la Investigación y el Conocimiento

DOI: 10.26820/recimundo/4.(4).octubre.2020.102-113

URL: http://recimundo.com/index.php/es/article/view/888

EDITORIAL: Saberes del Conocimiento

REVISTA: RECIMUNDO

ISSN: 2588-073X

TIPO DE INVESTIGACIÓN: Artículo de Revisión

CÓDIGo UNESCO: 32 Ciencias Médicas; 3201 Ciencias Clínicas

PAGINAS: 102-113

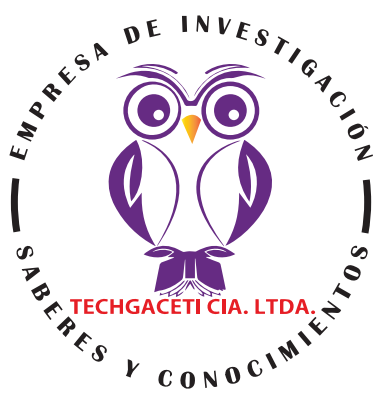

\title{
Megacolon tóxico. Tratamiento quirúrgico
}

Toxic megacolon. Surgical treatment

Megacólon tóxico. Tratamento cirúrgico

David Alexander Cepeda Arauz'; Edgar Josué Redrobán Tufiño2; Marielena Estefanía Murgueytio Salazar;

Daniela Alejandra Pozo Gualpa ${ }^{4}$

RECIBIDO: 10/07/2020 ACEPTADO: 26/08/2020 PUBLICADO: 15/10/2020

1. Médico de la Universidad Central del Ecuador; Hospital San Vicente de Paul, Médico Residente en el Área de Terapia Intensiva; Ibarra, Ecuador; alexnderdvid@gmail.com; (D) https://orcid.org/0000-0001-8197-4015

2. Médico General; Instituto Ecuatoriano de Seguridad Social; Riobamba; Ecuador jredroban@gmail.com; (iD https://orcid. org/0000-0002-1473-6268

3. Médica Cirujana; Residente Asistencial; Hospital de Especialidades Carlos Andrade Marín; Quito, Ecuador mmurgueytio@ udlanet.ec; (D) https://orcid.org/0000-0002-7047-0142

4. Médico General por la Universidad Central del Ecuador; Médico Residente de Cirugía Plástica en el Hospital de la Policia Quito N¹; Quito, Ecuador; danielitapoz015@gmail.com; iD hhttps://orcid.org/0000-0003-1109-8085

CORRESPONDENCIA

David Alexander Cepeda Arauz

alexnderdvid@gmail.com

Ibarra, Ecuador

ㄷ RECIMUNDO; Editorial Saberes del Conocimiento, 2020 


\section{RESUMEN}

El megacolon tóxico representa una complicación producto de afecciones principalmente inflamatorias o infecciosas del colon. Se asocia más comúnmente con la enfermedad inflamatoria intestinal (EII), es decir, colitis ulcerosa o enfermedad de Crohn ileocolónica. No obstante, ultimamente la epidemiología se ha desplazado hacia las causas infecciosas, específicamente debido a un aumento de la colitis asociada con la bacteria Clostridium Difficile posiblemente debido al uso extensivo de antibióticos de amplio espectro. Otras etiologías infecciosas importantes incluyen Salmonella, Shigella, Campylobacter, Citomegalovirus (CMV), Rotavirus, Aspergillus y Entameba. Con menos frecuencia, el megacolon tóxico se ha atribuido a colitis isquémica, colitis colagenosa o cáncer colorrectal obstructivo. La dilatación tóxica del colon también puede ocurrir en el síndrome urémico hemolítico (SUH) causado por Escherichiacoli enterohemorrágica o enteroagregativa (EHEC, EAEC o EAHEC). Los mecanismos fisiopatológicos que conducen a la dilatación tóxica del colon no se comprenden completamente. Las principales características del megacolon tóxico son signos de toxicidad sistémica y distensión colónica severa. El diagnóstico se realiza mediante evaluación clínica de toxicidad sistémica y estudios de imágenes que muestran dilatación colónica. El manejo del megacolon tóxico es una tarea interdisciplinaria que requiere una estrecha interacción de gastroenterólogos y cirujanos desde el principio. El momento óptimo de la cirugía para el megacolon tóxico puede ser un desafío. En el desarrollo del proceso investigativo se exponen los datos más recientes sobre la patogenia, la presentación clínica, el laboratorio y las modalidades de imágenes y se proporcionan algoritmos para un enfoque diagnóstico y terapéutico basado en evidencias.

Palabras clave: Megacolon tóxico, Colitis ulcerosa, Enfermedad de Crohn, Enfermedad inflamatoria intestinal, Clostridiumdifficile.

\section{ABSTRACT}

Toxic megacolon represents a complication resulting from mainly inflammatory or infectious conditions of the colon. It is most commonly associated with inflammatory bowel disease (IBD), that is, ulcerative colitis or ileocolonic Crohn's disease. However, lately epidemiology has shifted towards infectious causes, specifically due to an increase in colitis associated with the bacterium Clostridium Difficile possibly due to the extensive use of broad spectrum antibiotics. Other important infectious etiologies include Salmonella, Shigella, Campylobacter, Cytomegalovirus (CMV), Rotavirus, Aspergillus, and Entameba. Less commonly, toxic megacolon has been attributed to ischemic colitis, collagenous colitis, or obstructive colorectal cancer. Toxic dilation of the colon can also occur in hemolytic uremic syndrome (HUS) caused by enterohemorrhagic or enteroaggregative Escherichiacoli (EHEC, EAEC, or EAHEC). The pathophysiological mechanisms that lead to toxic dilation of the colon are not fully understood. The main characteristics of toxic megacolon are signs of systemic toxicity and severe colonic distention. Diagnosis is made by clinical evaluation of systemic toxicity and imaging studies showing colonic dilation. The management of toxic megacolon is an interdisciplinary task that requires close interaction of gastroenterologists and surgeons from the beginning. The optimal timing of surgery for toxic megacolon can be challenging. In the development of the investigative process, the most recent data on pathogenesis, clinical presentation, laboratory and imaging modalities are exposed and algorithms are provided for an evidence-based diagnostic and therapeutic approach.

Keywords: Toxic megacolon, Ulcerative colitis, Crohn's disease, Inflammatory bowel disease, Clostridium difficile.

\section{RESUMO}

O megacólon tóxico representa uma complicação resultante de condições principalmente inflamatórias ou infecciosas do cólon. É mais comumente associada à doença inflamatória intestinal (DII), ou seja, colite ulcerativa ou doença de Crohn ileocolônica. No entanto, ultimamente a epidemiologia mudou para causas infecciosas, especificamente devido ao aumento da colite associada à bactéria Clostridium Difficile, possivelmente devido ao uso extensivo de antibióticos de amplo espectro. Outras etiologias infecciosas importantes incluem Salmonella, Shigella, Campylobacter, Cytomegalovirus (CMV), Rotavirus, Aspergillus e Entameba. Menos comumente, o megacólon tóxico foi atribuído à colite isquêmica, colite colagenosa ou câncer colorretal obstrutivo. A dilatação tóxica do cólon também pode ocorrer na síndrome hemolítico-urêmica (SHU) causada por Escherichiacoli enterohemorrágico ou enteroagregativo (EHEC, EAEC ou EAHEC). Os mecanismos fisiopatológicos que levam à dilatação tóxica do cólon não são totalmente compreendidos. As principais características do megacólon tóxico são sinais de toxicidade sistêmica e distensão colônica severa. O diagnóstico é feito por avaliação clínica de toxicidade sistêmica e estudos de imagem que mostram dilatação do cólon. O manejo do megacólon tóxico é uma tarefa interdisciplinar que requer interação próxima de gastroenterologistas e cirurgiões desde o início. O momento ideal de cirurgia para megacólon tóxico pode ser desafiador. No desenvolvimento do processo investigativo, os dados mais recentes sobre patogênese, apresentação clínica, modalidades laboratoriais e de imagem são expostos e algoritmos são fornecidos para uma abordagem terapêutica e diagnóstica baseada em evidências.

Palavras-chave: Megacólon tóxico, Colite ulcerativa, Doença de Crohn, Doença inflamatória intestinal, Clostridium difficile. 


\section{Introducción}

El término " megacolon tóxico " denota una rara pero grave complicación potencialmente fatal de la inflamación del colon. Sus principales características son la evidencia radiográfica de distensión colónica total o segmentaria de $>6 \mathrm{~cm}$. A diferencia de otros tipos y causas de dilatación colónica como el síndrome de Ogilvie o la enfermedad de Hirschsprung, "el megacolon tóxico se define por la presencia adicional de toxicidad sistémica y la etiología inflamatoria o infecciosa de la enfermedad subyacente" (Guillermo \& Eduardo, 2018).

La prevalencia exacta del Megacolon Tóxico (MT) se desconoce, sin embargo, "ésta sigue aumentando con la edad la causa más común de ingreso hospitalario incluye la Ell $(51,6 \%)$, seguida de la septicemia $(10,2 \%)$ y las infecciones intestinales $(4,1 \%)$ " (Sergio \& Héctor, 2018). La incidencia de MT es mayor en pacientes con colitis ulcerosa (CU) en comparación con Enfermedad de Crohn (EC) (8-10\% en CU comparado con 2,3\% en EC). En contraste, Latella, Vernia, $\&$ Viscido, (2002) reportaron que su incidencia en EC es "mayor que en CU (4.4-6.3\% en EC comparado con $1-2.5 \%$ en $\mathrm{CU}$ ), adicionalmente la incidencia de TM por Clostridium difficile (C. diff) se estimó en 0,4 a 3\% antes de 1990; sin embargo, es del 4,3\% después de 1990".

El sexo femenino, la edad mayor de 40 años, la hipoalbuminemia, la acidosis y los niveles elevados de nitrógeno ureico en sangre se asocian con una alta mortalidad. Sin embargo, la tasa de mortalidad en pacientes con MT es variable. En años anteriores, "la tasa de mortalidad por MT era del $27 \%$ en los casos manejados médicamente, sin embargo, se evidencia más baja (19\%) en los casos manejados quirúrgicamente, logrando una notoria reducción de 0-2\% en pacientes con Ell" (Berman, Carling, \& Fitzgerald, 2008)
La identificación temprana y el manejo intensivo pueden haber contribuido a la reducción de la incidencia y la mortalidad de la MT en la ElI. Los autores Berman, Carling, \& Fitzgerald, (2008) exponen que "los pacientes con infección fulminante requieren intervención quirúrgica hasta en un $20 \%$ de los casos, con tasas de mortalidad entre el 35 y el $80 \% "$.

Es evidente que el padecimiento del megacolon es una patología a la que se le debe ir con prisa, esto para evitar complicaciones mayores. En el desarrollo de la investigación se realizará una revisión bibliográfica en aras de brindar información necesaria para futuras investigaciones que aporten significativamente a los temas que se relacionan con el megacolon toxico.

\section{Metodología}

Para el desarrollo de este proceso investigativo, se plantea como metodología la encaminada hacia una orientación científica particular que se encuentra determinada por la necesidad de indagar en forma precisa y coherente una situación, en tal sentido Davila, (2015) define la metodología "como aquellos pasos anteriores que son seleccionados por el investigador para lograr resultados favorables que le ayuden a plantear nuevas ideas" (p.66)

Lo citado por el autor, lleva a entender que el desarrollo de la acción investigativa busca simplemente coordinar acciones enmarcadas en una revisión bibliográfica con el fin de complementar ideas previas relacionadas Megacolon tóxico. Tratamiento quirúrgico a través de una revisión de literatura, para así finalmente elaborar un cuerpo de consideraciones generales que ayuden a ampliar el interés propuesto

\section{Tipo de Investigación}

Dentro de toda práctica investigativa, se precisan acciones de carácter metodológi- 
co mediante las cuales se logra conocer y proyectar los eventos posibles que la determinan. En este sentido, la presente investigación corresponde al tipo documental, definido por Castro (2016), "se ocupa del estudio de problemas planteados a nivel teórico, la información requerida para abordarlos se encuentra básicamente en materiales impresos, audiovisuales y / o electrónicos". (p.41).

En consideración a esta definición, la orientación metodológica incluye la oportunidad de cumplir con una serie de actividades inherentes a la revisión y lectura de diversos documentos, donde se encuentran ideas explicitas relacionadas con los tópicos encargados de identificar una característica inmersa en el estudio. Por lo tanto, se realizaron continuas interpretaciones con el claro propósito de revisar aquellas apreciaciones propuestas por diferentes investigadores en relación al tema de interés, para luego dar la respectiva argumentación a los planteamientos, en función a las necesidades encontradas en la investigación, apoyados en las herramientas tecnológicas para la búsqueda de trabajos con valor científico disponibles en la web que tenían conexión con el objetivo principal de la investigación.

\section{Fuentes Documentales}

El análisis correspondiente a las características que predomina en el tema seleccionado, llevan a incluir diferentes fuentes documentales encargadas de darle el respectivo valor científico y en ese sentido cumplir con la valoración de los hechos a fin de generar nuevos criterios que sirven de referencia a otros procesos investigativos. Para Castro,(2016) las fuentes documentales incorporadas en la investigación documental o bibliográfica, "representa la suma de materiales sistemáticos que son revisados en forma rigurosa y profunda para llegar a un análisis del fenómeno" (p.41). Por lo tanto, se procedió a cumplir con la lectura previa determinada para encontrar aquellos aspectos estrechamente vinculados con el tema, con el fin de explicar mediante un desarrollo las respectivas apreciaciones generales de importancia.

\section{Técnicas para la Recolección de la Infor- mación}

La conducción de la investigación para ser realizada en función a las particularidades que determinan a los estudios documentales, tiene como fin el desarrollo de un conjunto de acciones encargadas de llevar a la selección de técnicas estrechamente vinculadas con las características del estudio. Bolívar, (2015), refiere, que es "una técnica particular para aportar ayuda a los procedimientos de selección de las ideas primarias y secundarias". (p.71).

Tal como lo expresa, Bolívar, (2015) "Las técnicas documentales proporcionan las herramientas esenciales y determinantes para responder a los objetivos formulados y llegar a resultados efectivos" (p. 58). Es decir, para responder con eficiencia a las necesidades investigativas, se introdujeron como técnica de recolección el método inductivo, que hizo posible llevar a cabo una valoración de los hechos de forma particular para llegar a la explicación desde una visión general. El autor Bolívar, (2015) tambien expresa que las técnicas de procesamiento de datos en los estudios documentales "son las encargadas de ofrecer al investigador la visión o pasos que deben cumplir durante su ejercicio, cada una de ellas debe estar en correspondencia con el nivel a emplear" (p. 123). Esto indica, que para llevar a cabo el procesamiento de los datos obtenidos una vez aplicadas las técnicas seleccionadas, tales como: fichas de resumen, textual, registros descriptivos entre otros, los mismos se deben ajustar al nivel que ha sido seleccionado. 


\section{Resultados}

\section{Epidemiología}

Los datos precisos sobre la epidemiología del megacolon tóxico son escasos y los estudios más amplios se remontan a principios de la década de 1980. Además, la incidencia de megacolon tóxico varía según su etiología. Los estudios epidemiológicos anteriores se centraron principalmente en la incidencia de megacolon tóxico en la enfermedad inflamatoria intestinal (EII).

En un estudio retrospectivo Greenstein (como citó Sergio \& Héctor, 2018) revisaron 1236 pacientes con Ell ingresados en el hospital en un período de 1960 a 1979. En total, se identificaron 75 casos de megacolon tóxico (6\%). En el 10\% de los pacientes con colitis ulcerosa (CU) y en el 2,3\% con enfermedad de Crohn (EC), el trastorno se complicó con megacolon tóxico. En un estudio prospectivo más reciente, Richard, (2014) informó "megacolon tóxico en el $7,9 \%$ de los pacientes ingresados con CU".

Hasta finales de la década de 1990, "la incidencia de megacolon tóxico en pacientes con colitis pseudomembranosa (PMC) se informó entre $0,4 \%$ y $3 \%$. Durante la última década, sin embargo, se han observado cambios importantes en la epidemiología de las infecciones por Clostridiumdifficile (CDI)" (Richard, 2014)

El uso más frecuente de antibióticos de banda ancha y a la aparición de una cepa hipervirulenta de C. difficile (BI / NAP1 / 027) explican un aumento general del megacoIon tóxico asociado a C. difficile. Los cambios demográficos son un factor adicional en los cambios epidemiológicos. Los pacientes de 65 años o más tienen un mayor riesgo de desarrollar CDI y de cursos más graves de la enfermedad, incluido el megacolon tóxico. Los autores Cober \& Malani, (2009) examinaron retrospectivamente las historias clínicas de 70 pacientes con CDI mayores de 80 años ingresados en un centro de atención terciaria desde enero de 2006 hasta diciembre de 2006. Las complicaciones en esta población incluyeron tres casos de megacolon tóxico (4,3\%). En particular, también ha habido un aumento en el número de CDI adquiridos en la comunidad en los últimos años.

Además, un número cada vez mayor de pacientes que antes se consideraba de bajo riesgo, como las mujeres en el período periparto y los pacientes pediátricos, desarroIlan CDI adquirida en la comunidad. Hay un informe de caso de una "mujer embarazada con megacolon tóxico que se complica en una ICD grave. En ese caso, no se documentaron factores de riesgo tradicionales de CDI" (Zea, 2012). Megacolon tóxico o colon que complica la PMC se asocia con una tasa de mortalidad del $38 \%$ al $80 \%$.

Existe una sorprendente falta de datos fiables actuales sobre la epidemiología del megacolon tóxico. Por lo tanto, se requieren más estudios para tener en cuenta los avances terapéuticos, los factores etiológicos y los cambios demográficos en los últimos años.

\section{Etiología}

El megacolon tóxico se considera más comúnmente una complicación de la Ell, específicamente la $\mathrm{CU}$ y en mucho menor grado la CD. Sin embargo, a lo largo de las últimas décadas, la lista de factores etiológicos se amplió debido a una amplia gama de afecciones inflamatorias e infecciosas: colitis bacterianas como C. difficile, Salmonella, Shigella, y Cam-pylobacter así como viral (p. ej., citomegalovirus [CMV]) y parasitaria (Entameba) las infecciones pueden complicarse con la dilatación del colon. (Zea, 2012)

Otros factores etiológicos incluyen colitis isquémica, Enfermedad de Behcet y neoplasias como linfoma de colon y sarcoma 
de Kaposi. Existen informes de casos de "aspergilosis, colitis colagenosa, y rotavirus involucrado en megacolon tóxico. La dilatación tóxica del colon también puede acompañarsíndrome urémico hemolítico (SUH) causado por E. coli O157". (Helena \& María, 2017)

El megacolon tóxico también juega un papel en el paciente inmunodeprimido. Las in- fecciones por CMV y C. difficile son las causas predominantes de megacolon tóxico en pacientes con virus de inmunodeficiencia humana $(\mathrm{VIH})$ o SIDA. También se informa que la quimioterapia tóxica es una causa potencial de megacolon tóxico.

A continuación se presenta una tabla donde se describen los factores etiológicos del megacolon toxico.

Tabla 1. Factores etiológicos del megacolon tóxico

\begin{tabular}{|l|}
\hline Inflamatorio \\
Colitis ulcerosa \\
Enfermedad de Crohn \\
Enfermedad de Behc, et Infecciosa \\
Clostridium difficile \\
Salmonella, Shigella, Yersinia, Campylobacter, E. coli \\
Citomegalovirus, rotavirus \\
Entameba \\
Aspergilosis \\
Cryptosporidium \\
Isquemia \\
\hline Otras causas \\
Colitis colágena \\
Linfoma de colon \\
Sarcoma de Kaposi \\
Quimioterapia \\
\hline
\end{tabular}

Fuente: (Helena \& María, 2017).

\section{Patogénesis}

La fisiopatología precisa del megacolon tóxico no se comprende completamente. Sin embargo, "está bien establecida una asociación entre las condiciones inflamatorias del colon y la disminución de la contractilidad del músculo liso" (Alicia \& Silvia, 2019). La progresión de la CU no complicada al megacolon tóxico se estudia mejor hasta la fecha. Al estar limitados a la mucosa y las capas superficiales de la submucosa en la CU no complicada, los procesos inflamatorios penetran en la muscularis propia en el megacolon tóxico. La profundidad de la inflamación parece estar correlacionada con el grado de dilatación del colon. Además, se sabe que "varios mediadores inflamatorios tienen un efecto inhibidor sobre la motilidad colónica. El óxido nítrico (NO) como neurotransmisor no adrenérgico y no colinérgico clave induce la relajación del músculo liso del colon" (Alicia \& Silvia, 2019)

A continuación se presenta una tabla donde se observa el enfoque diagnostico del Megacolon toxico. 


\section{Presentación clínica e investigaciones de laboratorio}

El megacolon tóxico es un diagnóstico basado en signos clínicos de toxicidad sistémica en combinación con evidencia radiográfica de dilatación colónica. No parece haber predilección en ningún grupo de edad o género en particular. "Los pacientes con Ell tienen mayor riesgo de desarrollar megacolon tóxico en una etapa temprana de la enfermedad: hasta un 30\% de los pacientes se presentan dentro de los 3 meses posteriores al diagnóstico" (Sergio \& Héctor, 2018)

La historia de la enfermedad actual suele revelar signos y síntomas de colitis aguda antes del inicio de la dilatación colónica. Una historia cuidadosa de las posibles causas debe incluir una indagación sobre el diagnóstico previo de Ell, exposición a patógenos entéricos y medicación, específicamente esteroides, antibióticos y agentes antimotilidad (Tabla 2). En el examen físico, generalmente predominan los signos de toxicidad sistémica. La sensibilidad abdominal y la reducción de los ruidos intestinales son hallazgos frecuentes; los signos de peritonitis pueden indicar perforación colónica.

Es importante tener en cuenta el tratamiento con esteroides previo a la superficialidad, ya que puede enmascarar los síntomas de toxicidad. Los criterios clínicos más utilizados para el diagnóstico de megacolon tóxico fueron propuestos por Jalan en el 1969 (como citó Guillermo \& Eduardo, 2018). Tres de cuatro de los siguientes criterios principales son obligatorios para el diagnóstico clínico: fiebre, taquicardia, leucocitosis o anemia. Además, debe cumplirse uno de los siguientes criterios: deshidratación, alteración del nivel de conciencia, desequilibrio electrolítico o hipotensión.

Los estudios de laboratorio en pacientes con megacolon tóxico pueden presentar varias anomalías inespecíficas y reflejar la toxicidad del sistema y la gravedad de la colitis: la anemia y la leucocitosis con desviación a la izquierda es una característica común, así como un aumento de la velocidad de sedimentación globular y una proteína $\mathrm{C}$ reactiva elevada.

Los desequilibrios electrolíticos son uno de los principales criterios para el megacolon tóxico propuesto por Jalan. En particular, la hipopotasemia y la hipoalbuminemia se asocian con diarrea intensa, pérdida de volumen y un mal pronóstico en general. Se debe realizar un cribado fecal de patógenos para detectar hemocultivos de bacteriemia. La CDI se diagnostica mediante cultivo bacteriano o detección de toxina A y B en muestras de heces, por ejemplo, mediante ensayo inmunológico enzimático (EIA). (Faris, Blackmore, \& Haboubi, 2010)

\section{Estudios de imagen}

La detección de la dilatación del colon como característica crítica en el diagnóstico de megacolon tóxico es el dominio del examen radiológico. "Las características típicas que se observan en las radiografías simples de abdomen incluyen dilatación del colon de más de $6 \mathrm{~cm}$ y raramente hasta de 15 cm. Los autores Raúl \& José Luis, (2012) documentaron un "diámetro colónico medio de 9,2 cm, por lo general, el colon ascendente y el transverso son los más dilatados, otras características radiográficas incluyen niveles hidroaéreos colónicos y pérdida o alteración de la haustración colónica".

En una serie de casos Joaquín, (2014), los hallazgos ecográficos en cuatro pacientes con megacolon tóxico en la Ell fueron evaluado, "la ecografía reveló paredes colónicas delgadas con pérdida de haustracoli y aumento de diámetro (> $6 \mathrm{~cm}$ ) con contenido gaseoso similar a las características encontradas en las radiografías simples de abdomen". Esas características son inespe- 
cíficas y en el contexto individual puede ser difícil diferenciar entre megacolon tóxico y otras causas de dilatación colónica. Sin embargo, debido a la amplia disponibilidad de la ecografía, puede ser una valiosa herramienta de diagnóstico en el reconocimiento o sospecha de megacolon tóxico.

Las tomografías computarizadas fueron útiles para detectar signos de colitis grave, incluido el engrosamiento difuso de la pared del colon, haustras engrosadas con bandas alternas de alta y baja densidad (" signo del acordeón "), apariencia multicapa causada por diferentes densidades de submucosa edematosa y mucosa hiperémica (" signo de destino ") y varamiento pericólico. La detección adicional de dilatación colónica (diámetro transversal medio de $7,7 \mathrm{~cm}$, rango de 6 a $10 \mathrm{~cm}$ ) fue indicativa del desarrollo de megacolon tóxico (Joaquín, 2014). La TC fue más confiable para evaluar tanto la duración como la gravedad de la colitis y la presencia de dilatación del colon que las radiografías simples de abdomen. Además, La tomografía computarizada representa una herramienta importante en el diagnóstico de las complicaciones abdominales del megacolon tóxico, como la perforación o la pieloflebitis ascendente.

En la figura $N^{0} 1$ se observa corte trasversal de un asa intestinal en ecografia modo 8 en un paciente con EC. Con un transductor de alta resolución se pueden identificar en la pared 5 capas concéntricas: la capa hiperecogénica más interna corresponde a la interfase de la superficie mucosa y al contenido intestinal; capa hipoecogénica de la mucosa muscular; capa hiperecogénica central gruesa que corresponde a la submucosa; la capa hipoecogénica externa es la muscularis propia; y la serosa que es la capa hiperecogénica más externa.

Figura 1.

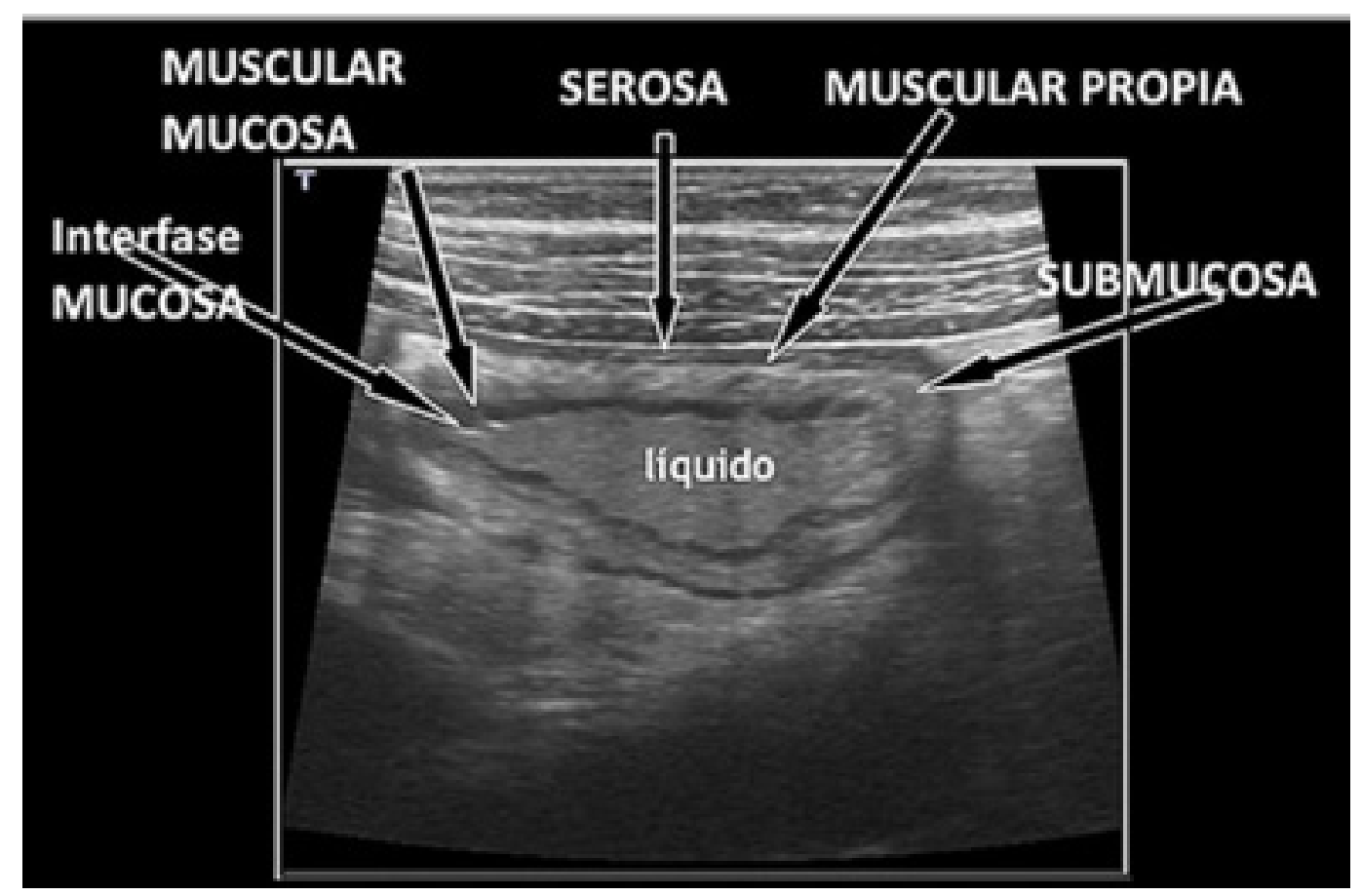

Fuente: (Tomás, 2015) 
Figura 2. Reconstrucción longitudinal del ciego $(\mathrm{C})$ e ileon terminal (IT) engrosados. Los valores máximos de sensibilidad de la ecografía corresponden a zonas de fácil acceso, como la afectación ileocecal.

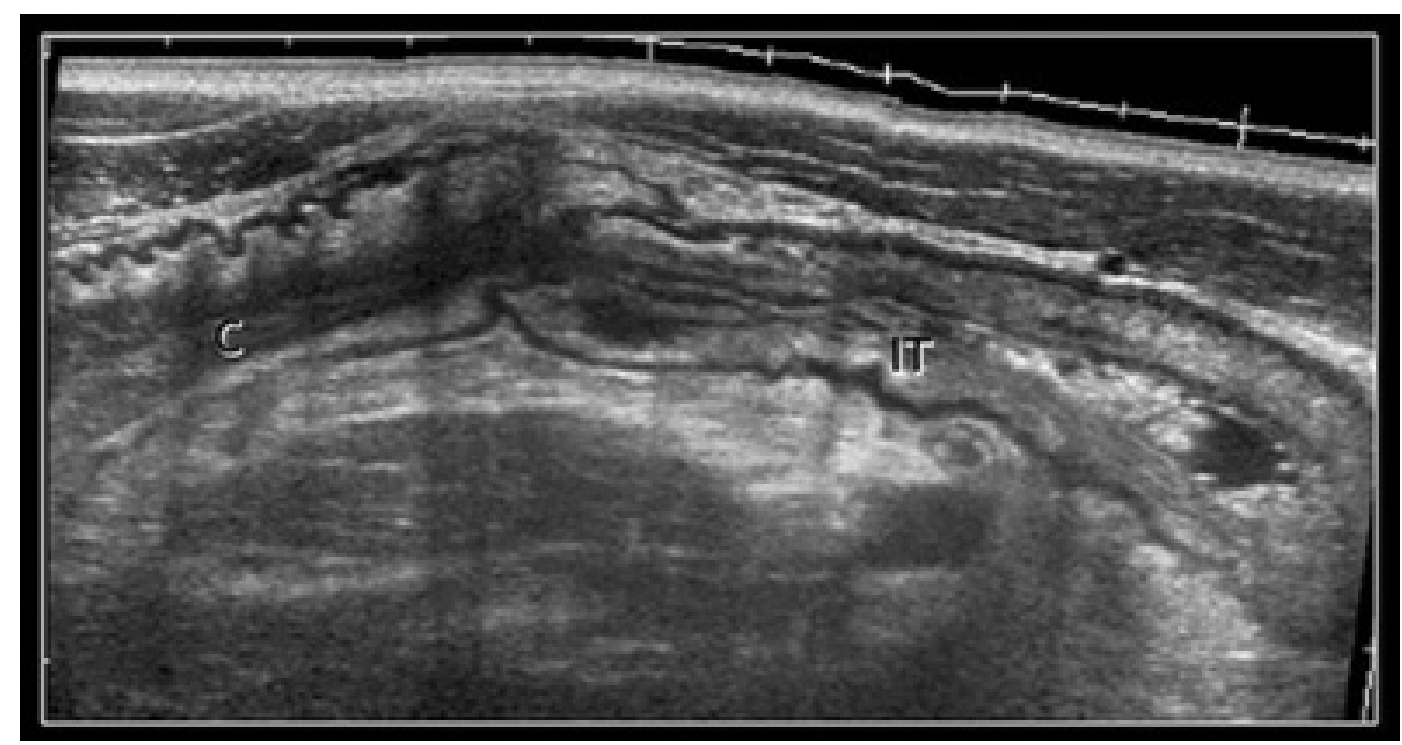

Fuente: (Tomás, 2015)

\section{Endoscopia}

En el contexto agudo de megacolon tóxico, la colonoscopia total conlleva un alto riesgo de perforación colónica y, por lo tanto, generalmente está contraindicada. Se considera que la "sigmoidoscopia limitada es valiosa para diferenciar las causas etiológicas, por ejemplo, para excluir el CMV o mediante la identificación de pseudomembranas en la colitis aguda por C. difficile" (Guillermo \& Eduardo, 2018). Por lo tanto, en el megacolon tóxico, el uso diagnóstico de la sigmoidoscopia flexible debe equilibrarse cuidadosamente con el alto riesgo deperforación. Si es necesario, el procedimiento debe realizarse únicamente con una mínima insuflación de aire.

\section{Administración}

La participación de expertos médicos y quirúrgicos es fundamental para un resultado favorable del megacolon tóxico.

Los principales objetivos del tratamiento del megacolon tóxico son el tratamiento de la causa subyacente, la atenuación de la colitis, el tratamiento de la toxemia y la evitación de complicaciones adicionales, específicamente la perforación intestinal. Es imprescindible una estrecha vigilancia médica y cuidados de apoyo. (Guillermo \& Eduardo, 2018)

Las anomalías en los niveles de electrolitos, especialmente la hipopotasemia, así como la deshidratación y la anemia pueden deteriorar la atonía o la dilatación del colon y, por lo tanto, deben controlarse y equilibrarse de manera agresiva. Las autoras Alicia \& Silvia, (2019) "considera que los medicamentos que afectan la motilidad del colon, por ejemplo, opiáceos, anticolinérgicos y antidiarreicos, deterioran el curso de la enfermedad y debe suspenderse inmediatamente". El reposo intestinal con nutrición parenteral total no se recomienda generalmente en la colitis aguda grave. También se sugiere el reposicionamiento de los pacientes como medio de descompresión intestinal mediante la redistribución del gas colónico hacia el colon distal y el recto. "Las técnicas de reposicionamiento incluyen maniobras de balanceo y una posición del paciente en decúbito prono rodilla-codo.sesenta y cinco Sin embargo, realizar esas maniobras en la 
rutina clínica puede ser impracticable" (Alicia \& Silvia, 2019)

La Ell se ha identificado como un factor de riesgo independiente de tromboembolismo venoso. Helena \& María, (2017) exponen "un riesgo de 3,6 veces mayor de tromboembolismo en pacientes con Ell en comparación con los sujetos de control". En consecuencia, la profilaxis de la trombosis venosa profunda con heparina en dosis bajas juega un papel importante en el tratamiento médico básico del megacolon tóxico causado por la Ell.

\section{Terapia medica}

Todavía hay una falta de estudios controlados que investiguen el manejo médico del megacolon tóxico. Los regímenes comúnmente aceptados se basan en el tratamiento de la causa subyacente, estudios retrospectivos y opiniones de expertos. El diagnóstico de megacolon tóxico no es una indicación absoluta para un curso de tratamiento quirúrgico. Sin embargo, para evitar la colectomía, el tratamiento médico debe realizarse de forma agresiva y oportuna.

El pilar de la terapia médica para pacientes con megacolon tóxico causado por CU son los esteroides intravenosos en dosis altas. El tratamiento con esteroides debe iniciarse de inmediato y no debe retrasarse por los resultados microbiológicos pendientes. "Se recomienda una dosis diaria de $400 \mathrm{mg}$ de hidrocortisona (100 mg cada 6 horas) o 60 mg de metilprednisolona administrada por vía intravenosa durante unos 5 días" (Guillermo \& Eduardo, 2018)

No hay beneficio en dosis mucho más altas de esteroides o extensión de la terapia. La idea de que el uso de esteroides aumenta el riesgo de perforación no está respaldada por datos clínicos.

Sin embargo, los signos clínicos de perforación del colon, así como los síntomas de to- xicidad, pueden quedar enmascarados por el uso de esteroides en dosis altas. Además, la tasa de fracaso de los esteroides intravenosos en pacientes con colitis grave es del $20 \%$ al $40 \%$. Para evitar retrasos en el momento de la cirugía necesaria, la terapia con esteroides debe reevaluarse con regularidad. Además, no se deben administrar esteroides una vez que se ha establecido una causa exclusivamente infecciosa de megacolon tóxico. (Latella, Vernia, \& Viscido, 2002)

No hay datos que respalden un beneficio de los aminsalicilatos orales. Por lo tanto, la sulfasalazina y el 5-aminosalicilato (5-ASA) no juegan ningún papel en el tratamiento del megacolon tóxico causado por la Ell. Aunque algunos lo utilizan en el contexto de la CU grave, no hay estudios controlados que investiguen el uso de ciclosporina A o el anticuerpo monoclonal infliximab en megacolon tóxico.

\section{Cirugía}

Los métodos quirúrgicos propuestos previamente en el tratamiento del megacolon tóxico incluyen la resección por colectomía subtotal con ileostomía terminal, proctocolectomíatotal o el método Turnbull. Los autores Attila \& Paulo, (2016) expresan que "a principios de la década de 1970, Turnbull introdujo un método de descompresión quirúrgica del colon". En resumen, el procedimiento tenía como objetivo reducir el riesgo perioperatorio de perforación y preparar a los pacientes para una colectomía definitiva en un momento posterior mediante la descompresión del colon en tres ubicaciones diferentes (orificios nasales) y la realización de una ileostomía.

\section{Terapia médica vs. Cirugía}

El momento de la cirugía en el megacolon tóxico sigue siendo motivo de controversia. Eludir la necesidad de cirugía constituye el objetivo principal de todo tratamiento médi-

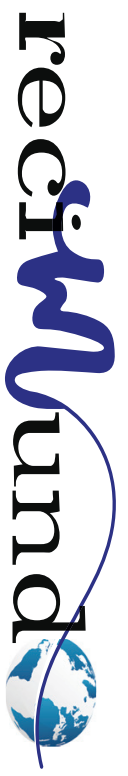


co. Sin embargo, "el retraso de la terapia quirúrgica conlleva el riesgo de complicaciones como perforación intestinal o síndrome compartimental abdominal que conllevan un mal pronóstico" (Alicia \& Silvia, 2019). Las indicaciones absolutas para la colectomía incluyen perforación, sangrado incontrolable del colon o recto y deterioro clínico general.

La controversia sobre el momento adecuado de la cirugía ilustra la importancia del tratamiento interdisciplinario. "Se debe buscar una consulta quirúrgica de alto nivel desde el principio en el curso de la enfermedad y se deben evaluar diariamente tanto la continuación del tratamiento médico como la posible indicación de colectomía" (Alicia \& Silvia, 2019). Los signos de complicaciones o deterioro deben ser una indicación de tratamiento quirúrgico. El megacolon tóxico todavía tiene una alta morbilidad y mortalidad. Se puede evitar una estrecha coordinación de la experiencia médica y quirúrgica con retrasos potencialmente fatales en el tratamiento correcto.

\section{Conclusión}

Durante el desarrollo del proceso investigativo se observó que el Megacolon tóxico (MT) es una condición que se aborda mejor a través de la combinación de esfuerzos del equipo médico y quirúrgico que trabaja con el paciente. La terapia médica es el tratamiento de primera línea en la mayoría de los casos, pero las medidas objetivas de mejora deben discutirse antes de iniciar el tratamiento.

Es importante tomar en cuenta que si el paciente manifiesta signos de hemorragia, peritonitis o perforación, la cirugía está indicada de forma urgente, colectomía e ileostomía subtotal / totales el procedimiento de elección en la mayoría de los casos, y un procedimiento laparoscópico El enfoque se favorece potencialmente cuando sea posible y factible.
No obstante, con los continuos avances en la terapia médica, las técnicas quirúrgicas y el cuidado posoperatorio, los resultados quirúrgicos son significativamente mejores que hace 50 años en el paciente que requiere cirugía urgente y de emergencia para la colitis fulminante. A pesar de esto, la morbilidad sigue siendo característica y la atención no solo a la técnica intraoperatoria sino también a los cuidados posoperatorios es fundamental.

Aunque hay centros que atienden un gran volumen de casos en pacientes con CU, los pacientes con colitis fulminante, con frecuencia, se presentan en hospitales con menos experiencia en su atención. Como se indicó anteriormente, la consideración del estado médico de los pacientes individuales y los aspectos relacionados con la calidad de vida es importante para elegir el procedimiento quirúrgico más apropiado.

\section{Bibliografía}

Alicia, S., \& Silvia, N. (2019). Manejo de la enfermedad inflamatoria intestinal. revisión y algoritmos de tratamiento. Acta Gastroenterol Latinoam

Berman, L., Carling, T., \& Fitzgerald, T. (2008). Definición de la terapia quirúrgica para la colitis pseudomembranosa con megacolon tóxico. . J Clin Gastroen-terol. , 476-480.

Faris, B., Blackmore, A., \& Haboubi, N. (2010). Review of medical and surgical management of Clostridium difficile infection. . TechColoproctol., 14:97-105.

Guillermo, F., \& Eduardo, C. (2018). Colitis ulcerosa, una mirada por dentro. Ciencias Médicas de Pinar del Río. vol. 22(3), 476-485.

Helena, F., \& María, M. (2017). Linfoma primario del intestino delgado: reporte de un caso y revisión de la literatura. Revista Colombiana de Gastroenterología, 32(1), 65-71. doi:https://doi. org/10.22516/25007440.132

Joaquín, P. (2014). Utilidad de la ecografía abdominal en el diagnóstico y seguimiento de la enfermedad inflamatoria intestinal. Rev Esp Enferm Dig (Madrid), 106(6), 395-408. 
Latella, G., Vernia, P., \& Viscido, A. (2002). Gl distension in severe ulcera-tive colitis. Am J Gastroenterol. , 1169-1175.

Richard, R. (11 de Abril de 2014). Vivir con colitis ulcerosa. Recuperado el 3 de Octubre de 2020, de https://www.crohnscolitisfoundation.org/sites/ default/files/legacy/vivir-con-la-colitis-ulcerosa.pdf

Sergio, S., \& Héctor, C. (2018). Megacolon tóxico de origen idiopático: reporte de caso. Asociaciones Colombianas de Gastroenterología.

Tomás, R. (30 de Mayo de 2015). Diagnóstico y seguimiento de los pacientes con enfermedad inflamatoria intestinal por ecografía. Recuperado el 3 de Octubre de 2020, de http://endoinflama- toria.com/diagnostico-y-seguimiento-de-los-pacientes-con-enfermedad-inflamatoria-intestinal-por-ecografia/

Zea, J. W. (2012). Enfermedad asociada a Clostridium difficile: prevalencia y diagnóstico por laboratorio. Infectio, 16(4), 211-222. Obtenido de https://www.sciencedirect.com/science/article/pii/ S0123939212700164

\section{CITAR ESTE ARTICULO:}

Cepeda Arauz, D. A., Redrobán Tufiño, E. J., Murgueytio Salazar, M. E., \& Pozo Gualpa, D. A. (2020). Megacolon tóxico. Tratamiento quirúrgico. RECIMUNDO, 4(4), 102-113. Recuperado a partir de http://recimundo.com/index.php/ es/article/view/888 